\title{
PHYSICOCHEMICAL CHARACTERIZATION OF BLUEBERRY (VACCINIUM SPP.) JUICES FROM 55 CULTIVARS GROWN IN NORTHERN SPAIN
}

\author{
R. Rodríguez Madrera*, B. Suárez Valles, A. Campa Negrillo \\ and J.J. FERREIRA FERNÁNDEZ
}

Regional Agrifood Research and Development Service (SERIDA), E-33300 Villaviciosa (Asturias). Spain

(Received: 11 September 2018; accepted: 10 December 2018)

\begin{abstract}
Blueberry (Vaccinium spp.) cultivation has experienced a notable increase both for its good organoleptic characteristics and the nutritional and functional properties of this berry. The aim of this study was the physicochemical characterization of blueberry juices obtained from 55 blueberry cultivars grown under the same environmental conditions for 2-4 years. The results provide a broad and robust database, both for the number of cultivars and the periods of monitoring thereof, in order to cover different aspects of blueberry processing, and more specifically, production of juices. Blueberries belonging to $V$. virgatum cultivars showed the higher values of total anthocyanin content, total phenolic content, antioxidant activity, soluble solids, and $\mathrm{pH}$, and $\mathrm{V}$. corymbosum cultivars the higher level of titratable acidity. Results also showed a high variability among cultivars. Observed variations can be used in plant breeding and classification of blueberry cultivars, at least, at the species level.

Keywords: blueberry, Vaccinium spp., antioxidant activity, total anthocyanin content, total phenolic content, discriminant analysis
\end{abstract}

The blueberry is a shrub native to North America and Europe including more than 400 species of the genus Vaccinium spp., family Ericaceae. The main cultivated species around the world are of American origin: V. corymbosum L., or highbush blueberries; V. virgatum Aiton, or rabbiteye; V. angustifolium Aiton, or lowbush blueberries; V. macrocarpum Aiton, or American cranberry. Hybrid cultivars from interspecific crosses are also grown (RetAmales \& HANCOCK, 2012).

Blueberry not suitable for fresh consumption is most frequently processed into juice, which is considered as a source of bioactive compounds (BRAmBILLA et al., 2008). Several studies have been focused on the impact of juice processing methods on its composition (Brambilla et al., 2008; Chen et al, 2014; HowArD et al., 2016), nevertheless, data about composition of the juices from the available cultivars are limited and refer to a few ones. In this sense, provide information on the chemical composition of juices obtained from the commercially available varieties will help choosing the appropriate cultivars to obtain products with the desired characteristics.

The aim of the study was the physicochemical characterization of blueberry juices from a set of 55 cultivars, maintained under the same agronomic conditions, at least for two years. The study covered different parameters (soluble solids, titratable acidity, $\mathrm{pH}$, total anthocyanin content, total phenolic content, and antioxidant activity) with interest both in the manufacture of blueberry juices and from a nutritional and functional point of view.

\footnotetext{
* To whom correspondence should be addressed.

Phone: +34 985890066; fax: +34 985891854; e-mail: rrodriguez@serida.org
} 


\section{Materials and methods}

\subsection{Plant material}

Fifty-nine accessions (Table 1) belonging to 55 cultivars (cvs) maintained in the SERIDA field collection (Villaviciosa, Asturias, Spain; 43 $29^{\prime} 01^{\prime \prime} \mathrm{N}, 5^{\circ} 26^{\prime} 11^{\prime \prime} \mathrm{W}$; elevation $6.5 \mathrm{~m}$ ) were used. The collection includes two plants per accession spaced at $1 \times 2.5 \mathrm{~m}$ and watered by drip irrigation. Fruit were harvested at maturity. Two harvests were carried out per year for each cultivar.

\subsection{Juice extraction}

Samples (100 g) were milled using a commercial blender (Moulinex Model JU200), centrifuged $(5000 \mathrm{~g}, 20 \mathrm{~min})$, and the juices were frozen until analysed.

\subsection{Physicochemical analyses}

Soluble solids (SS) were determined using a refractometer MA871 (Milwaukee Instruments Inc, Rocky Mount, NC, USA). Titratable acidity (TA) was determined by titrating $10 \mathrm{ml}$ of sample and $10 \mathrm{ml}$ of type I water with $0.1 \mathrm{~N}$ sodium hydroxide at $\mathrm{pH} 8.1$. The $\mathrm{pH}$ was measured in a Basic $20 \mathrm{pH}$-meter (Crison Instruments, Barcelona, Spain). Total anthocyanin content (TAC) was estimated by the $\mathrm{pH}$ differential method (WrOLSTAD, 1976). Total phenolic content (TPC) by Folin's method and antioxidant activity (AA) by DPPH method were determined according to DiÑEIRO GARCíA and co-workers (2009).

\subsection{Statistical analyses}

A two-way analysis of variance, cultivar and harvest year, taking harvest year as random variable, was carried out to detect significant differences in the composition of blueberry juices among cultivars.

A two-way analysis of variance, species and harvest year, taking harvest year as random variable, was carried out to detect significant differences in the composition of blueberry juices between species V. corymbosum and V. virgatum.

Principal Component Analysis (PCA) was employed to visualize the data structure, and Linear Discriminant Analysis (LDA) was used for classification purposes.

The software used was SPSS 15.0 (SPSS Inc, Chicago, IL, USA).

\section{Results and discussion}

Table 1 shows the assigned species obtained from the passport database, the harvest data, and the results of the physicochemical analysis of the blueberry juices.

The cultivars included the two main cultivated species (V. corymbosum and V. virgatum) and covered a wide range of harvest period, from June until the end of September.

The composition of the blueberry juices showed significant differences $(\mathrm{P}<0.0001)$ among the cultivars for all parameters studied (Table 1). 


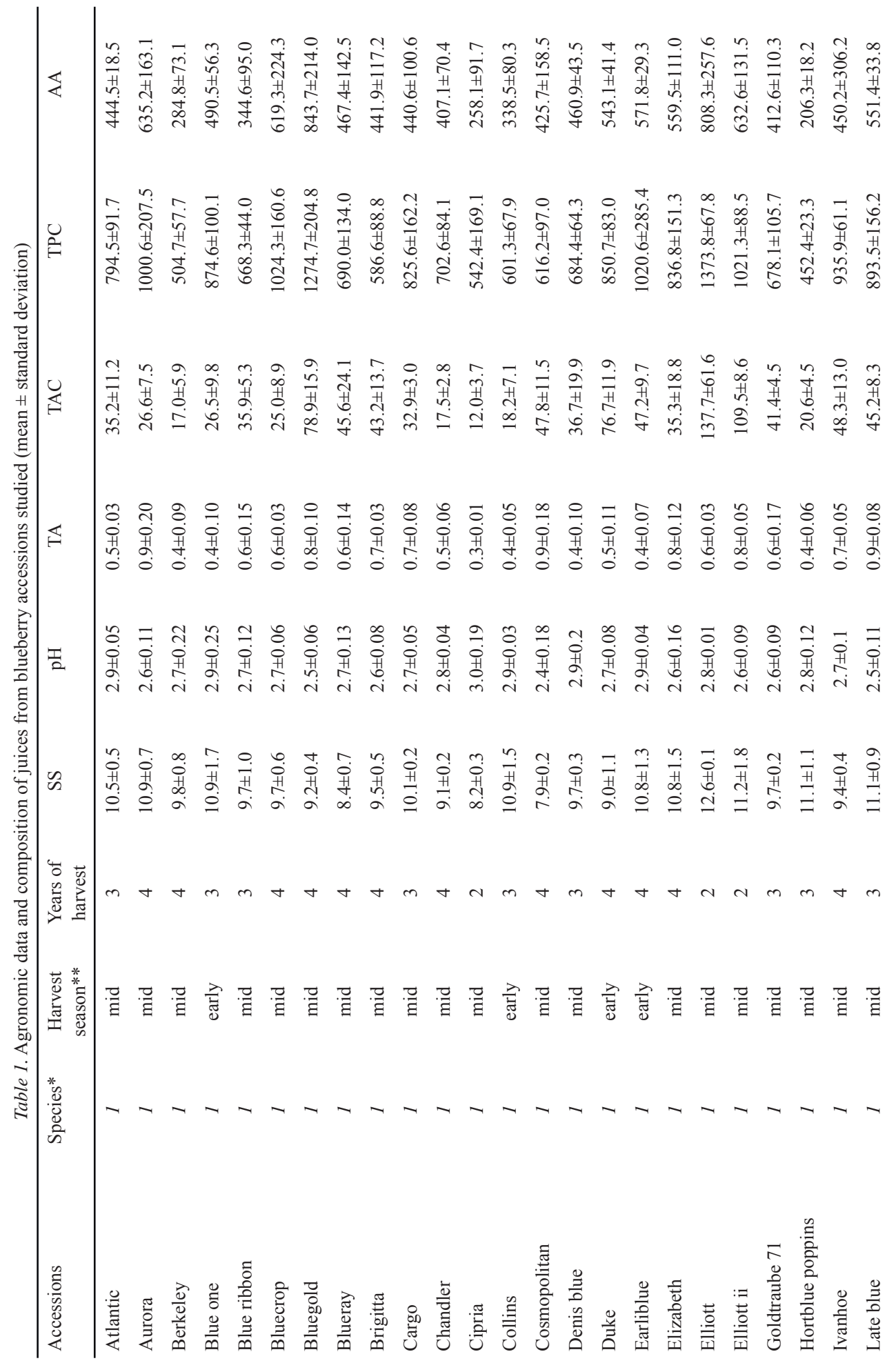


RODRÍGUEZ MADRERA et al.: CHARACTERIZATION OF BLUEBERRY JUICES

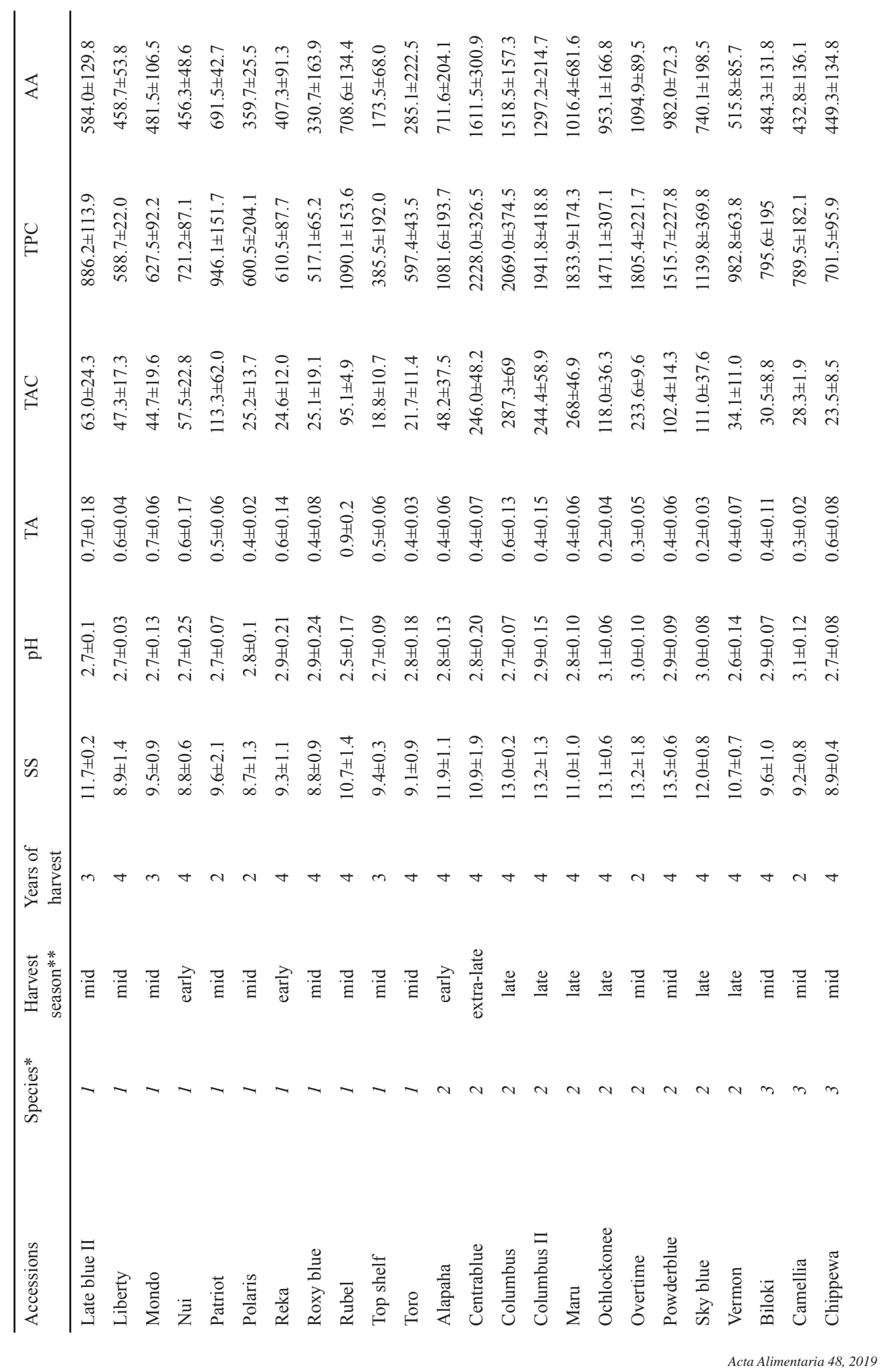




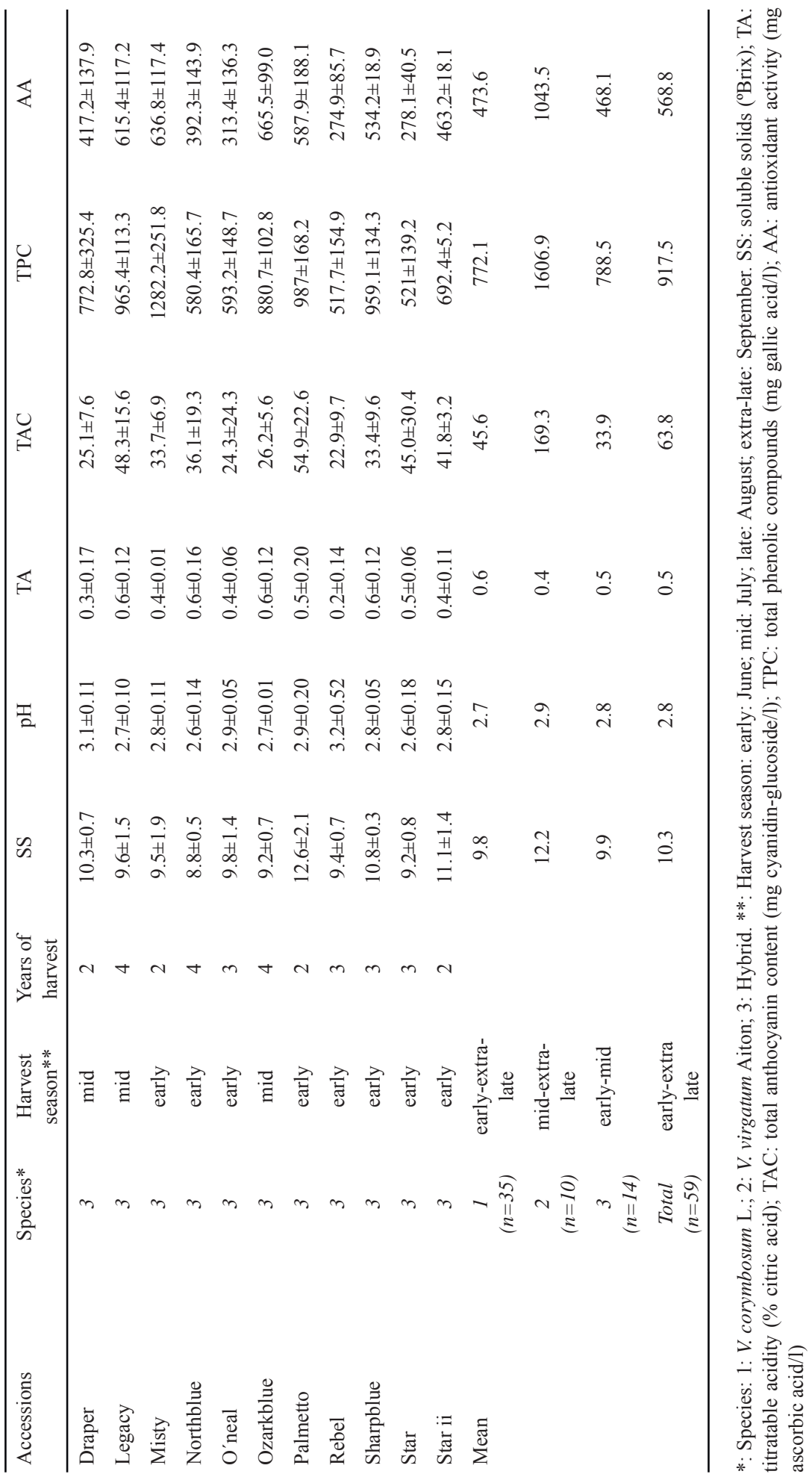


TA ranged between $0.2 \%$ (cvs Ochlockonee, Rebel, and Sky blue) and 0.9\% citric acid (cvs Aurora, Late blue, Rubel, and Cosmopolitan), with an average value of $0.5 \%$. These values are in accordance with the data reported by SKUPIEN (2006), while KIM and co-workers (2013) reported values of TA between 0.8 and 3.6\%. The differences among the data provided by other research groups for the same cultivar (SKUPIEN, 2006; KIM et al., 2013; ZorENC et al., 2016) can be due to the ripening process, the environment or the interaction genotypeenvironment.

Cultivars Cosmopolitan and Rebel presented the most extreme $\mathrm{pH}$ values, 2.4 and 3.2, respectively. Only 4 of the 59 accessions presented $\mathrm{pH}$ mean values higher than 3.0 (cvs Rebel, Ochlockonee, Camelia, and Drapper), while only in 3 of the 45 samples studied by KIM and co-workers (2013) were the $\mathrm{pH}$ values equal to or less than 3.0. In this sense, it is important to note that lower $\mathrm{pH}$ levels can facilitate the stability of anthocyanins in blueberry juices (Howard et al., 2016).

The sugar content, expressed as \% soluble solids (SS), showed values between 7.9 and $13.5^{\circ}$ Brix for the cvs Cosmopolitan and Powderblue, respectively. These values agree with the ranges described by SKUPIEN (2006), although some authors have published results of SS differing by more than $25 \%$ for the same cultivars (KIM et al., 2013).

TA and SS contribute to organoleptic characteristics of juices. BETT-GARBER and coworkers (2015) detected significant correlations between TA and the descriptors 'sour' and 'throat burn' in blueberry juices, and between SS and sweet taste, which would allow estimating differences in the properties of the juices from the data in Table 1.

Ranges between $10-33$ to SS/TA ratio and 2.25-4.25 to $\mathrm{pH}$ were suggested as indicators of good quality for blueberry (BEAUDRY, 1992). According to these parameters, 48 of 55 cultivars studied showed optimum values, only excepting the cvs Ochlockonee, Overtime, Powerblue, Sky blue, Draper, and Rebel (ratio >33) and Cosmopolitan (ratio <10).

Polyphenols contribute prominently to the antioxidant activity of blueberries, considered one of the most valuable functional properties of this fruit (BEATTIE et al., 2005). TPC ranged between $385.5 \mathrm{mg}$ gallic acid/l (cv Topshelf) and $2228.0 \mathrm{mg} \mathrm{GAE} / \mathrm{l}$ (cv Centrablue), with an average content in the collection of $917.5 \mathrm{mg}$ GAE/l. TAC ranged between 12.0 and 287.3 mg cyanidin 3-glucoside/1 for cvs Cipria and Columbus, respectively, with an average value of $63.8 \mathrm{mg}$ cyanidin-glucoside/l juice. TPC and TAC values agree with values reported in blueberry juices (KALt et al., 2000; Chen et al, 2014), although BRAmBiLla and co-workers (2008) described higher anthocyanin content for some of the cultivars studied in this work. The high variability in TAC within each cultivar can be due to various environmental and agronomic factors. ZORENC and co-workers (2016) also detected, for the same cultivar, differences in TAC of around $29 \%$ between the first and third harvest date, and KALT and coworkers (2001a) observed the inter annual variation can reach up to 2.4 times for both abiotic and biotic reasons. On the other side, TAC and TPC reported in the whole fruit are around ten times higher (Stevenson \& ScAlzo, 2012; KIM et al., 2013) and with TAC/TPC ratios in the range $0.15-1.1$. The analysed juices showed TAC/TPC ratios between $0.02-0.15$ for the cvs Cipria and Maru, respectively. In this sense, it should be noted that the determination of these families of compounds in the berry is done after extraction in methanolic medium, which favors the extraction of phenolic compounds in general and anthocyanins in particular (KIM et al., 2013).

Antioxidant activity (AA) showed differences among the cultivars of almost an order of magnitude between the cultivars with the highest and lowest antioxidant activities: 1611.5 $\mathrm{mg}$ ascorbic acid/l (cv Centrablue) and $173.5 \mathrm{mg}$ ascorbic acid/l (cv Top shelf). TAC and TPC 
correlated positively between them and with AA in the samples evaluated $\left(\mathrm{r}_{\text {TACTPC }}=0.893\right.$ and $\mathrm{P}<0.0001, \mathrm{r}_{\text {TAC/AA }}=0.896$ and $\mathrm{P}<0.0001 ; \mathrm{r}_{\text {TPC/AA }}=0.971$, and $\mathrm{P}<0.0001$ ), which highlights the large contribution of these chemical families to antioxidant activity.

Significant differences were detected between the species $V$. corymbosum and $V$. virgatum $(\mathrm{P}<0.01)$ for all variables studied. The phenolic compounds are mainly located in the skin of the fruit, so the juices from $V$. virgatum cultivars, producers of small fruit and therefore with a greater skin/fruit ratio, are those that presented the highest concentration, twofold higher, of TPA, TPC, and AA (Table 1). Stevenson and Scalzo (2012) found a significant correlation between fruit size and total phenolic content $(\mathrm{r}=-0.50)$ and total anthocyanins $(\mathrm{r}=-0.48)$, and KALT and co-workers (2001b) detected higher content of anthocyanins in the species V. angustifolium versus V. corymbosum; however, these authors could not establish a relationship between fruit size and anthocyanin content. Moreover, $V$. corymbosum cultivars showed a higher TA, with a higher average content of 50\% (Table 1), while for the $V$. virgatum species a higher $\mathrm{pH}$ value was detected. Likewise, the average content of SS was significantly higher (23\%) for the species V. virgatum, revealing important differences not only at the cultivar level but also at the species level.

PCA on the data matrix showed two significant components (eigenvalues $>1$ ) that accounted for $87.6 \%$ of the variance (Fig. 1). Figure 2 displays the loadings of each variable in these axes. V. virgatum cultivars, with higher values of SS, TAC, TPC, and AA, are located on the right side of the principal component 1 ( $\mathrm{PC} 1$ ), while samples of $V$. corymbosum, with higher values of TA, are located on the left side of this axis. The projection of the scores obtained for the hybrid cultivars placed these individuals in the zone of the V. corymbosum cultivars (Fig. 1), which shows the similarity of the berries produced by the hybrids and the taxons of the species V. corymbosum for the variables studied.

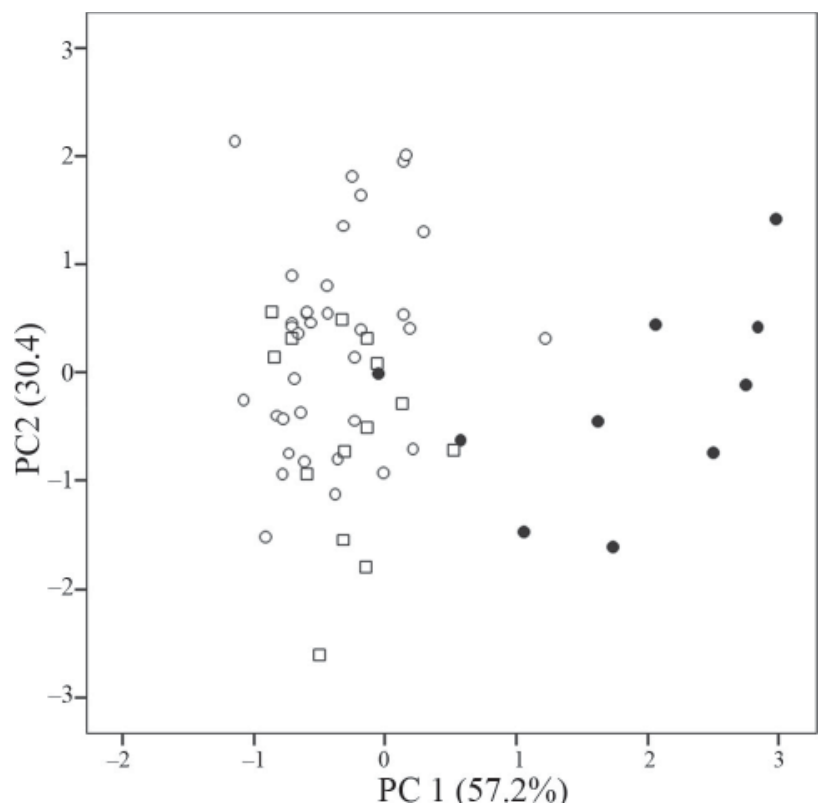

Fig. 1. Projection of the 59 accessions of blueberry on the plane formed by the 2 principal components (PC). ०: V. corymbosum; •: V. virgatum; $\square$ : Hybrid 


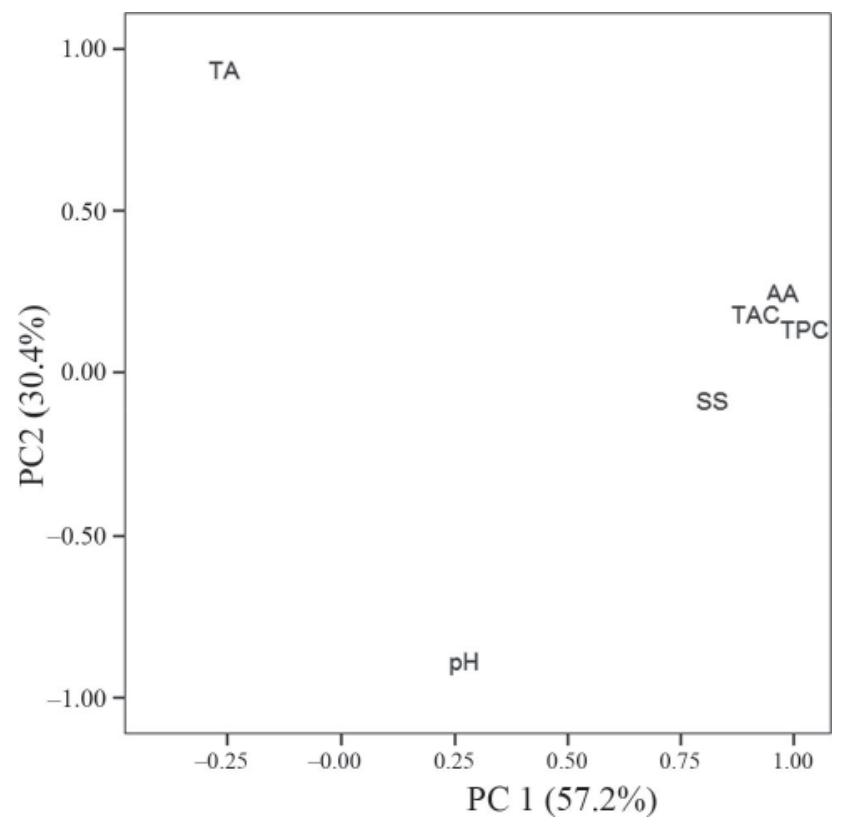

Fig. 2. Loadings plot for first and second components

SS: Soluble solids ( ${ }^{\circ}$ Brix); TA: titratable acidity (\% citric acid); TAC: total anthocyanin content (mg cyanidinglucoside/l); TPC: total phenolic compounds (mg gallic acid/l); AA: antioxidant activity (mg ascorbic acid/l)

Finally, the chemical variables were used for classification and predictive purposes, at the species level, through a stepwise LDA in order to know the most discriminant variables. To build the model, the variables TAC and AA were rejected, while the most important variables were TPC and TA. In this way, a discriminant function (F) was computed:

$$
\mathrm{F}=-0.472 \mathrm{~A}_{1}+9.368 \mathrm{~A}_{2}+11.894 \mathrm{~A}_{3}-0.003 \mathrm{~A}_{4}-24.599
$$

with centroids $\mathrm{C}_{1}=1.166$ ( $\mathrm{V}$. corymbosum) and $\mathrm{C}_{2}=-4.082$ ( $\mathrm{V}$. virgatum), where $\mathrm{A}_{1}$ : $\mathrm{SS} ; \mathrm{A}_{2}$ : $\mathrm{pH} ; \mathrm{A}_{3}$ : TA and $\mathrm{A}_{4}$ : TPC. Classification hits, and prediction hits by leave-one-out cross validation, were $100 \%$ for all cultivars of species $V$. corymbosum and $V$. virgatum. When the discriminant function was used to classify the hybrid cultivars, all of them, except Misty (classified as $V$. virgatum) were classified as $V$. corymbosum. In this sense, CAMPA and FERREIRA (2018) have shown the genetic proximity between the hybrid cultivars of this study and the species $V$. corymbosum.

\section{Conclusions}

The blueberry cultivars studied covered a wide genetic diversity and a wide range for harvest period. The study provides a broad and robust database, both for the number of cultivars and the periods of monitoring thereof, in order to cover different aspects of blueberry production and more specifically of obtaining juices. Blueberries belonging to $V$. virgatum cultivars showed the higher values of TAC, TPC, AA, SS, and $\mathrm{pH}$, and V. corymbosum cultivars the 
higher level of TA. The parameters studied showed a high variability among cultivars as a result of abiotic and biotic conditions, which highlights the need to conduct characterization studies in time. The parameters analysed in this study could be used to classify the cultivars at the species level through LDA.

This work was supported by the grant RTA2013-00076 from INIA - Ministerio Economía y Competitividad (Spanish Government) and co-financing from FEDER funds. Ana Campa is paid by INIA-CCAA (DR13-0222). Authors thank M. Bueno, J.A. Poladura, and F. Díaz for their technical assistance.

\section{References}

Beattie, J., Crozier, A. \& Duthie, G.G. (2005): Potential health benefits of berries. Curr. Nutr. Food Sci., 1, 71-86. Beaudry, R. (1992): Blueberry quality characteristics and how they can be optimized. Annu. Rep. Mich. State Hortic. Soc., 122, 140-145.

Bett-Garber, K., Lea, J.L., Watso, M.A., Grimm, C.C., Lloyd, S.W., Neaulieu, J.C., Stein-Chisholm, R.E., Andrzejewski, B.P. \& Marshall, D.A. (2015): Flavor of fresh blueberry juice and the comparison to amount of sugar, acids, anthocyanidins, and physicochemical measurements. J. Food Sci., 80, S818-S827.

Brambilla, A., Lo Scalzo, R., Bertolo, G. \& Torreggiani, D. (2008): Steam-blanched highbush blueberry (Vaccinium corymbosum L.) juice: phenolic profile and antioxidant capacity in relation to cultivar selection. $J$. Agr. Food Chem., 56, 2643-2648.

CAmpa, A. \& Ferreira, J.J. (2018): Genetic diversity assessed by genotyping by sequencing (GBS) and for phenological traits in blueberry cultivars. Plos One, 13, e0206361.

Chen, J., Tao, X.Y, Sun, A.D., Wang, Y., Liao, X.-J., Li, L.-N. \& Zhang, S. (2014): Influence of pulsed electric field and thermal treatments on the quality of blueberry juice. Int. J. Food Prop., 17, 1419-1427.

Diñeiro García, Y., Suárez Valles, B. \& Picinelli Lobo, A. (2009): Phenolic and antioxidant composition of byproducts from the cider industry. Food Chem., 117, 731-738.

Howard, L.R., Brownmiller, C., Mauromoustakos, A. \& Prior, R.L. (2016): Improved stability of blueberry juice anthocyanins by acidification and refrigeration. J. Berry Res., 6, 189-201.

Kalt, W., McDonald, J.E. \& Donner, H. (2000): Anthocyanins, phenolics, and antioxidant capacity of processed lowbush blueberry products. J. Food Sci., 65, 390-393.

Kalt, W., Howell, A., Duy, J.C., Forney, C.F. \& McDonald, J.E. (2001a): Horticultural factors affecting antioxidant capacity of blueberries and other small fruit. HortTechnology, 11, 523-528.

Kalt, W., Ryan, D.A., Duy, J.C., Prior, R.L., Ehlenfeldt, M.K. \& Van der Kloet, S.P. (2001b): Interspecific variation in anthocyanins, phenolics, and antioxidant capacity among genotypes of highbush and lowbush blueberries (Vaccinium section cyanococcus spp.). J. Agr. Food Chem., 49, 4761-4767.

Kim, J.G., Kim, H.L., KIm, S.J. \& PARK, K-S. (2013): Fruit quality, anthocyanin and total phenolic contents, and antioxidant activities of 45 blueberry cultivars grown in Suwon, Korea. J. Zhejiang Univ. Sci. B., 14, 793-799.

Retamales, J.B. \& Hancock, J.F. (2012): Blueberries: Crop production science in horticulture. $N^{o} 21$. Wallinford, UK: CABI publishing. 136 pages.

SKupIEN, K. (2006): Chemical composition of selected cultivars of highbush blueberry fruit (Vaccinium corymbosum L.). Folia Hortic., 18, 47-56.

Stevenson, D. \& Scalzo, J. (2012): Anthocyanin composition and content of blueberries from around the world. J. Berry Res., 2, 179-189.

Wrolstad, R.E. (1976): Color and pigment analyses in fruit products (Report No. 624). Oregon State University. Agricultural Experiment Station. 17 pages.

Zorenc, Z., Veberic, R., Stampar, F., Koron, D. \& Mikulic-Petkovsek, M. (2016): Changes in berry quality of northern highbush blueberry (Vaccinium corymbosum L.) during the harvest season. Turk. J. Agric. For., 40, 855-864. 